\title{
ANÁLISE DOS RESULTADOS DO ÚLTIMO LAUDO CITOPATOLÓGICO DE PACIENTES COM PAPANICOLAOU EM ATRASO
}

Nathanael de Souza Maciel ${ }^{1}$

Francisco Jardsom Moura Luzia ${ }^{1}$

Diego da Silva Ferreira ${ }^{1}$

Maria Charlianne de Lima Pereira Silva

Davide Carlos Joaquim ${ }^{1}$

Leilane Barbosa de Sousa ${ }^{1}$

\author{
https://orcid.org/0000-0002-5088-011X \\ https://orcid.org/0000-0002-8386-6103 \\ https://orcid.org/0000-0002-6314-5405 \\ https://orcid.org/0000-0002-3258-6793 \\ https://orcid.org/0000-0003-0245-3110 \\ https://orcid.org/0000-0003-0266-6255
}

Objetivo: Analisar os resultados do último laudo citopatológico de pacientes com o exame Papanicolaou em atraso. Metodologia: Estudo documental, retrospectivo, com abordagem quantitativa, realizado no município de Redenção-CE, no período de abril a setembro de 2018. Foram explorados 660 prontuários de mulheres com idade entre 25 e 64 anos. Para análise foram utilizadas medidas de tendência central e frequências absolutas e relativas. Resultados: $O$ número de mulheres que nunca haviam realizado o exame Papanicolaou foi de $44,7 \%$ e as que haviam realizado, mas estavam em atraso, foi de $55,3 \%$. A presença de células metaplásicas foi menos frequente (38,6\%). Entre agentes infecciosos, houve prevalência dos resultados sugestivos de Gardnerella vaginalis e Mobiluncus (14,9\%), Cândida sp (3,9\%) e Trichomonas vaginalis (1,1\%). Os laudos indicaram presença de alterações celulares pré-malignas e malignas. Conclusão: Há um grande número de mulheres com o exame Papanicolaou em atraso e uma deficiência na qualidade dos exames, indicada pela ausência de tecido metaplásico. Isto deve motivar a busca ativa das pacientes para interromper a cadeia de transmissão de Infecções Sexualmente Transmissíveis e tratar lesões no colo do útero precocemente, reduzindo as chances de progressão.

Descritores: Neoplasias do Colo do Útero; Teste de Papanicolau; Programas de Rastreamento; Atenção Primária à Saúde: Enfermagem.

\section{ANALYSIS OF THE RESULTS OF THE LAST CYTOPATHOLOGICAL EXAM OF PATIENTS WITH DELAYED PAP SMEAR}

Objective: To analyze the results of the last cytopathological report of patients with delayed Pap smear. Methodology: Documentary, retrospective study with quantitative approach, conducted in the city of Redenção-CE, from April to September 2018. Were explored 660 medical records of women aged 25 to 64 years. For analysis, measures of central tendency and absolute and relative frequencies were used. Results: The number of women who had never had the Pap smear was $44,7 \%$, and those who had been submitted but were delayed were $55,3 \%$. The presence of metaplastic cells was less frequent (38,6\%). Among infectious agents, there was a prevalence of suggestive results of Gardnerella vaginalis and Mobiluncus (14,9\%), Candida sp $(3,9 \%)$ and Trichomonas vaginalis (1,1\%). The reports indicated the presence of premalignant and malignant cellular alterations. Conclusion: There are a large number of women with delayed Pap tests and a deficiency in the quality of the tests, indicated by the absence of metaplastic tissue. This should motivate the active search of patients to interrupt the chain of transmission of Sexually Transmitted Infections and to treat cervical lesions early, reducing the chances of progression.

Descriptors: Uterine Cervical Neoplasms, Papanicolaou Test; Mass Screening; Primary Health Care; Nursing.

\section{ANÁLISIS DE LOS RESULTADOS DEL ÚLTIMO EXAMEN CITOPATOLÓGICO DE PACIENTES CON PAPANICOLAOU EN RETRASO}

Objetivo: Analizar resultados del último examen citopatológico de pacientes con Papanicolaou en retraso. Metodología: Estudio documental, retrospectivo, con enfoque cuantitativo, realizado en la ciudad de Redenção-CE, de abril a septiembre de 2018. Fueron explorados 660 prontuarios de mujeres de 25 a 64 años. Para el análisis, se utilizaron medidas de tendencia central, frecuencias absolutas y relativas. Resultados: El número de mujeres que nunca se habian hecho la prueba de Papanicolaou fue del $44,7 \%$, y las que se sometieron, pero estában en retraso, fueron 55,3\%. La presencia de células metaplásicas fue menos frecuente (38,6\%). Entre los agentes infecciosos, hubo una prevalencia de resultados sugestivos de Gardnerella vaginalis y Mobiluncus (14,9\%), Candida sp (3,9\%) y Trichomonas vaginalis (1,1\%). Los informes indicaron presencia de alteraciones celulares premalignas y malignas. Conclusión: Hay una gran cantidad de mujeres con pruebas de Papanicolaou retrasadas y una deficiencia en la calidad de los exámenes, indicada por la ausencia de tejido metaplásico. Esto debería motivar la búsqueda activa de pacientes para interrumpir la cadena de transmisión de las infecciones de transmisión sexual y para tratar las lesiones cervicales temprano, reduciendo las posibilidades de progresión.

Descritores: Neoplasias del Cuello Uterino; Prueba de Papanicolaou; Tamizaje Masivo; Atención Primaria de Salud; Enfermería.

¿Universidade da Integração Internacional da Lusofonia Afro-Brasileira, Redenção, CE, Brasil.

Autor Correspondente: Nathanael de Souza Maciel Email: nathanael.souza.inf@gmail.com Recebido: 17/11/19 Aceito: 07/4/20 


\section{INTRODUÇÃO}

O câncer do colo do útero (CCU) é uma das mais acentuadas ameaças à vida das mulheres, sendo uma das principais causas de morte por câncer nas mulheres de todo o mundo. ${ }^{1}$ Com uma estimativa de 570.000 casos e 311.000 mortes em 2018 em todo o mundo, esta doença classifica-se como o quarto câncer mais frequentemente diagnosticado e o quarto com maior mortalidade. ${ }^{2}$ No período de 2014 a 2018, estima-se que o Brasil foi o sexto país mais incidente do mundo (1l.733 casos), o sétimo mais prevalente (33.460 casos), sendo o quinto país com mais óbitos em todo o mundo, totalizando 5.244 mortes. $^{3}$

Com a finalidade de decrescer a incidência de CCU no Brasil, o exame Papanicolaou é tido como a estratégia predominante de rastreamento e detecção precoce do $\mathrm{CCU}$, evidenciado e admitido mundialmente como seguro e eficiente. ${ }^{4}$ Este exame consiste na coleta do material da endocérvice e ectocérvice para análises citopatológicas cérvico-vaginal e microbiológica, deve ser realizado anualmente e, se o mesmo apresentar dois resultados negativos consecutivos, a periodicidade do exame deverá ser de três em três anos. ${ }^{5}$

O rastreamento do $\mathrm{CCU}$ e suas lesões precursoras por meio do exame Papanicolaou é essencial, pois viabiliza detectar e tratar precocemente as lesões precursoras do câncer antes da sua evolução para uma doença invasiva. Ademais, é possivel detectar outros achados no exame Papanicolaou, como alterações celulares benignas (reativas ou reparativas), inflamação sem identificação de agente, achados microbiológicos (Lactobacillus sp.; Cocos, e outros Bacilos), vaginites, infecções sexualmente transmissiveis, dentre outras manifestações clínicas. ${ }^{4}$

A Atenção Primária à Saúde (APS) se adequa como a via preferencial de acesso ao Sistema de Saúde e como centro de comunicação da Redes de Atenção à Saúde, sistematizando - cuidado e ordenando as ações e serviços ofertados na rede. É atribuição da APS desenvolver ações de saúde que incorporem promoção, prevenção, proteção, diagnóstico, tratamento e reabilitação, valendo-se de práticas de cuidado integrado e gestão qualificada. ${ }^{6}$ Nesta perspectiva, o elemento mais importante no âmbito da APS para que se obtenha considerável redução da incidência e da mortalidade por CCU é o alcance de uma cobertura elevada da população delimitada como alvo desse problema. ${ }^{4}$

Entretanto, como ainda não há programas efetivamente organizados de rastreamento do CCU no Brasil, não há controle das mulheres que realizam os exames e nem da periodicidade com que o fazem. ${ }^{5}$ Neste âmbito, é importante que os profissionais da equipe multiprofissional de saúde, sobretudo os enfermeiros, estejam atentos e contribuam para o rastreamento, prevenção e controle do CCU, adotando diferentes estratégias, como a identificação de pacientes com o referido exame em atraso, a interpretação e análise dos resultados dos respectivos laudos citopatológicos, a fim de verificar alguma alteração com possibilidade de progressão que reforce a necessidade da realização de busca ativa.

O enfermeiro possui grande relevância por manter um dos maiores vínculos com as pacientes, por isso necessita conhecer a realidade local da área onde atua para que melhor planeje ações e implemente-as, afim de reduzir as taxas de morbimortalidade por $\mathrm{CCU}$, possibilitando a efetividade da estruturação da prevenção. ${ }^{7}$ Diante disso, acredita-se que este estudo poderá contribuir para o diagnóstico situacional acerca da cobertura do CCU e da caracterização dos laudos de pacientes com o Papanicolaou em atraso, bem como instigar reflexões nos profissionais de enfermagem, com o intuito de aperfeiçoar as estratégias de busca ativa na prevenção do CCU.

Nesta perspectiva, o objetivo desta pesquisa consistiu em analisar os resultados do último laudo citopatológico de pacientes com o exame Papanicolaou em atraso.

\section{MÉTODO}

\section{Tipo de estudo}

Estudo documental, descritivo e retrospectivo, com abordagem quantitativa.

\section{Participantes da pesquisa}

A amostra não probabilística desse estudo correspondeu a 660 prontuários. Foram incluídos na amostra os prontuários de mulheres que tinham entre 25 e 64 anos de idade, com atividade sexual já iniciada e que haviam realizado o exame Papanicolaou há mais de três anos, após dois exames normais consecutivos realizados com um intervalo de um ano, ou que tinha realizado apenas um exame normal há mais de um ano. ${ }^{4}$ Foram excluidos os laudos com ausência de informações, como a data de realização do último exame.

\section{Local do estudo}

O estudo foi realizado em quatro unidades de atenção primária à saúde (UAPS) da zona urbana do município de Redenção, interior do Estado do Ceará, no período de abril a setembro de 2018 .

\section{Coleta de dados}

Os dados foram obtidos por meio da análise, nos prontuários das pacientes, das seguintes variáveis: data de nascimento, data da realização do último exame, epitélios presentes na amostra, microbiota identificada e análise celular. Foi construída uma planilha no software Microsoft Office Excel 2016 para organizar e armazenar os dados obtidos. 


\section{Procedimentos de análise dos dados}

A análise dos dados foi realizada por meio do programa de acesso livre EpiInfo, versão 7.2.1.0 (CDC, Atlanta - EUA). Foram aplicados os procedimentos da estatística descritiva nos dados de todas as mulheres que estavam em atraso com o exame de Papanicolaou. Para análise das variáveis numéricas, foram utilizadas medidas de tendência central, ao passo que foram apresentadas frequências absolutas e relativas das variáveis categóricas.

\section{Procedimentos éticos}

Para atender aos critérios éticos, a pesquisa foi submetida ao Comitê de Ética em Pesquisa da Universidade da Integração Internacional da Lusofonia Afro-Brasileira e aprovado sob o parecer de $n^{\circ}$ 3.033.528 e CAAE 91003518.8.0000.5576. Por se tratar de um estudo retrospectivo de inferência documental dos prontuários, dispensou-se a assinatura do Termo de Consentimento Livre e Esclarecido.

\section{RESULTADOS}

A idade média das mulheres atendidas na UABS foi de $41( \pm 10,51)$ anos. Na Tabela 1, constam informações relacionadas à situação das pacientes acerca da realização do exame Papanicolaou.

Dentre os 660 prontuários analisados, 365 (55,3\%) eram de mulheres que haviam realizado o exame, mas estavam com o mesmo em atraso. Neste grupo, a faixa etária mais frequente foi a 35 a 44 anos, com 126 (34,5\%) dos registros. Os dados revelam que 295 $(44,7 \%)$ mulheres nunca haviam realizado o exame, com maior frequência na faixa etária de 25 a 34 anos. A realização do exame preventivo demonstrou decrescer com o aumento da idade da mulher, conforme se pode notar pela diminuição do número de atendimentos a partir da faixa etária de 55 a 64 $\operatorname{anos}(14,4 \%)$.

Tabela 1 - Distribuição das mulheres conforme realização do exame Papanicolaou e faixa etária. Redenção, CE, Brasil, 2019

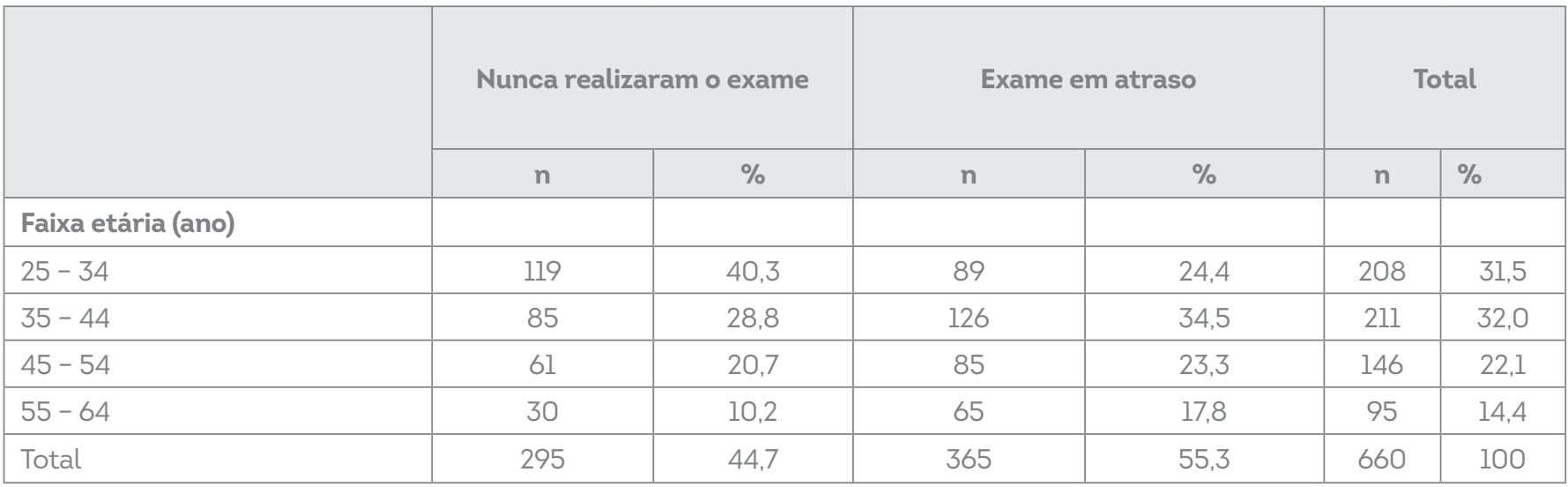

Dos prontuários de mulheres que haviam sido submetidas ao Papanicolaou, 308 (84,4\%) registros continham o tipo de epitélio no laudo. A Tabela 2 indica os epitélios representados na amostra, informação obrigatória nos laudos citopatológicos.
As células escamosas foram as que apresentaram registros mais frequentes, aparecendo em 306 (99,4\%) dos laudos que continham registros, sucedido por células glandulares (59,1\%). A presença de células metaplásicas foi a menos frequente $(38,6 \%)$.

Tabela 2 - Distribuição das mulheres conforme epitélios identificados no exame Papanicolaou e faixa etária. Redenção, CE, Brasil, 2019

\begin{tabular}{|c|c|c|c|c|c|c|c|c|c|c|}
\hline & \multicolumn{2}{|c|}{$25-34$} & \multicolumn{2}{|c|}{$35-44$} & \multicolumn{2}{|c|}{$45-54$} & \multicolumn{2}{|c|}{$55-64$} & \multirow[b]{2}{*}{$\mathbf{n}$} & \multirow[b]{2}{*}{$\%$} \\
\hline & $\mathbf{n}$ & $\%$ & $\mathbf{n}$ & $\%$ & $\mathrm{n}$ & $\%$ & $\mathrm{n}$ & $\%$ & & \\
\hline Escamoso & 77 & 25,2 & 102 & 33,3 & 73 & 23,9 & 54 & 17,6 & 306 & 100 \\
\hline Glandular & 46 & 25,3 & 56 & 30,8 & 50 & 27,5 & 30 & 16,5 & 182 & 100 \\
\hline Metaplásico & 25 & 21,0 & 46 & 38,7 & 23 & 21,0 & 23 & 19,3 & 119 & 100 \\
\hline
\end{tabular}


A Tabela 3 apresenta os achados microbiológicos constituintes da microbiota vaginal normal.

Os Bacilos apresentaram maior frequência, de Lactobacilos sp.

Tabela 3 - Distribuição das mulheres conforme faixa etária e microbiota identificada nos laudos. Redenção, CE, Brasil, 2019

\begin{tabular}{|l|c|c|c|c|c|c|c|c|c|c|}
\hline Bacilos & & & & & & & & & & \\
\hline Sim & 55 & 21,9 & 87 & 34,7 & 60 & 23,9 & 49 & 19,5 & 251 & 100 \\
\hline Não & 32 & 28,8 & 39 & 35,1 & 24 & 21,6 & 16 & 14,4 & 111 & 100 \\
\hline Cocos & & & & & & & & & & \\
\hline Sim & 19 & 15,6 & 47 & 38,5 & 27 & 22,1 & 29 & 23,8 & 122 & 100 \\
\hline Não & 68 & 28,3 & 79 & 32,9 & 57 & 23,8 & 36 & 15,0 & 240 & 100 \\
\hline Lactobacilos & 7 & 25,9 & 12 & 44,4 & 7 & 25,9 & 1 & 3,7 & 27 & 100 \\
\hline Sim & 80 & 23,9 & 114 & 34,0 & 77 & 23,0 & 64 & 19,1 & 335 & 100 \\
\hline Não & & & & & & & & & \\
\hline
\end{tabular}

A Tabela 4 apresenta alterações em relação a presença de algum agente infeccioso.

Os resultados sugestivos de Gardnerella vaginalis e Mobiluncus corresponderam a 54 (14,9\%) registros, tendo maior incidência (19,5\%) na faixa etária de 25 a 34 anos e um decréscimo inversamente proporcional à idade. Gardnerella vaginalis apareceu em 20 (5,5\%) registros e Cândida sp esteve presente em 14 (3,9\%) dos laudos, ambas com prevalência acentuada na faixa etária de 55 a 64 anos. Trichomonas vaginalis apresentou quatro $(1,1 \%)$ dos registros e Chlamydia um registro. Além desses, 11 (3,0\%) laudos apresentaram registros de citólise, aparecendo na tabela como outros.

Os laudos ainda indicaram a presença de alterações celulares, sendo um registro de células escamosas atípicas de significado indeterminado, possivelmente não neoplásicas, um registro de lesão intraepitelial escamosa de alto grau (NIC II/III) e um registro de lesão intraepitelial de alto grau não podendo excluir microinvasão ou carcinoma epidermoide invasor.

Tabela 4 - Distribuição dos agentes infecciosos presentes no exame Papanicolaou segundo a faixa etária. Redenção, CE, Brasil, 2019

\begin{tabular}{|l|c|c|c|c|c|c|c|c|c|c|}
\hline Bacilos Supr & 17 & 31,5 & 19 & 35,2 & 12 & 22,2 & 6 & 11,1 & 54 & 100 \\
\hline Gardnerella & 6 & 30,0 & 7 & 35,0 & 3 & 15 & 4 & 20 & 20 & 100 \\
\hline Candida & 4 & 28,6 & 5 & 35,7 & 3 & 21,4 & 2 & 14,3 & 14 & 100 \\
\hline Trichomonas & 1 & 25 & 0 & 0 & 1 & 25 & 2 & 50 & 4 & 100 \\
\hline Chlamydia & 0 & 0 & 1 & 100 & 0 & 0 & 0 & 0 & 1 & 100 \\
\hline Outros & 3 & 27,3 & 3 & 27,3 & 5 & 45,4 & 0 & 0 & 11 & 3,0 \\
\hline
\end{tabular}




\section{DISCUSSÃO}

Com a criação do exame Papanicolaou na década de 1940, a citologia cervical ganhou um método robusto e de baixa complexidade para o rastreamento do câncer do colo do útero. ${ }^{8}$ Com amplo conhecimento da biologia celular e com inúmeras ferramentas de triagem e prevenção, o CCU pode ser detectado em um estágio inicial suficiente para ser curável.?

A realização periódica de exame de prevenção do $\mathrm{CCU}$ é a estratégia mais adotada para o rastreamento desse tipo de câncer. Recomenda-se o rastreamento citopatológico em mulheres de 25 a 64 anos de idade e o intervalo entre os exames deve ser de três anos, após dois exames anuais consecutivos negativos. ${ }^{4}$ Essa faixa etária está em consonância com as recomendações da Organização Mundial de Saúde. ${ }^{10}$

Apesar das recomendações do Ministério da Saúde do Brasil, muitas mulheres nunca realizaram o exame preventivo. A desinformação, questões sociais, os medos e os tabus, são fatores negativos que as distanciam do exame.7 Além disso, é notório que a citologia diagnóstica poderia ajudar a facilitar o diagnóstico precoce do câncer do colo do útero, otimizando a triagem de mulheres jovens com idade entre 20 e 29 anos. ${ }^{11}$ Portanto, o profissional enfermeiro, através da criação de vínculos com a paciente, pode ser um agente na comunicação entre usuárias e serviços de saúde, oportunizando a prevenção de CCU por meio da adesão ao rastreamento.

Estudo descritivo, retrospectivo e transversal realizado em UAPS do Município de Juazeiro do Norte-CE, constatou que as mulheres na faixa etária 25 a 34 anos foram as que mais realizaram o exame Papanicolaou, ${ }^{12}$ o que contrasta com o presente estudo. Na pesquisa retrocitada, evidenciouse que as mulheres na faixa etária de 55 a 64 anos foram as que menos realizaram exame Papanicolaou, resultado similar aos achados desta análise. Resultado semelhante foi também encontrado em um inquérito domiciliar realizado com 250 mulheres adscritas numa Unidade de Saúde da Família do município de Sinop - MT, onde a faixa etária de 45 a 59 anos foi a que menos realizou o exame. ${ }^{13}$

É fato que o país ainda não dispõe de um sistema de informação de base populacional, item importantíssimo para um rastreamento organizado. ${ }^{5} \mathrm{O}$ rastreio oportunista, que ocorre em contextos clínicos e depende da iniciativa da mulher ou do seu médico, deve ser desencorajado. Tais atividades são muitas vezes caracterizadas por alta cobertura em partes selecionadas da população que são examinadas com demasiada frequência, coexistindo com baixa cobertura em outros grupos populacionais com menos condições socioeconômicas, resultando em eficácia limitada e custobenefício ruim. Um programa de triagem bem organizado deve alcançar alta aceitação, cobertura da população, deve garantir e demonstrar boa qualidade em todos os niveis de atenção à saúde. ${ }^{14}$

Os resultados da análise dos epitélios se assemelham aos achados de outro estudo, ${ }^{12}$ onde registrou-se a maior prevalência do epitélio escamoso (80,9\%), seguido do epitélio glandular (49,1\%) e o metaplásico (7,4\%). Nessa perspectiva, é válido salientar que a presença exclusiva de células escamosas deve ser analisada, sob pena de não propiciar à mulher todos os benefícios da prevenção do câncer do colo do útero. ${ }^{4}$ Assim, é muito favorável que os profissionais de saúde observem para a representatividade da junção escamocolunar nos esfregaços cervicovaginais.

A presença do epitélio metaplásico tem sido sugerida como indicador de qualidade do exame, pelo fato de este epitélio se situar no local onde se encontra a quase totalidade dos cânceres do útero. ${ }^{4}$ Nesse sentido, é importante que sejam criadas oportunidades de educação e capacitação continuadas que promovam a melhoria dos profissionais e das técnicas para análises laboratoriais, afim de evitar erros desde a coleta até a interpretação, o que poderia acarretar na liberação de resultados errôneos, comprometendo o tratamento e até a sobrevida do paciente. ${ }^{15}$

A análise microbiológica encontrada nesse estudo corrobora achados de um estudo realizado no Estado do Maranhão.16 que identificou bacilos (52,8\%), cocos $(45,5 \%)$ e Lactobacilus sp (32,6\%). Achados semelhantes foram também encontrados em outra pesquisa realizada, que revelou o predomínio bacilos, cocos e lactobacilus sp. ${ }^{17}$ Estes microrganismos não caracterizam infecção que necessita de tratamento, a menos que a paciente mencione alguma sintomatologia, uma vez que representam aspectos naturais da microbiota vaginal. ${ }^{18-19}$

Os resultados referentes aos agentes infecciosos encontrados neste estudo se assemelham a outro estudo, ${ }^{20}$ onde a cândida sp e a gardnerella vaginallis/mobilluncus, apareceram 4,45\% e 12,74\%, respectivamente. Nas infecções sexualmente transmissiveis, tais como tricomoníase e clamídia, foram obtidos, respectivamente, 2,48\% e 0,33\%, valores semelhantes aos encontrados neste estudo. Desta forma, torna-se relevante o papel dos programas de saúde sexual e reprodutiva, fomentando seu engajamento em projetos de educação em saúde para prevenção de doenças. ${ }^{21}$

As alterações celulares encontradas nos laudos salientam a necessidade de focar em educação permanente dos profissionais da saúde, sobretudo enfermeiros, para que sejam capacitados sobre o manejo do CCU. O conteúdo do treinamento dos profissionais pode incluir informações sobre métodos de rastreamento do CCU, como interpretar resultados anormais e encaminhar os pacientes para os outros níveis de atenção à saúde. Além disso, o acompanhamento por 
meio de referência e contrarreferência da usuária poderiam ser melhor explicados e consequentemente fortalecidos. ${ }^{22}$

A informação em saúde é uma estratégia de triagem transversal, seja por meio de ações educativas, visando cooperar com a construção e adoção de uma postura assistencial positiva ou através da análise de registros que permitam identificar mulheres em situação de vulnerabilidade e que garantam maior qualidade às ações profissionais. Assim, as ações de vigilância são essenciais para o efetivo manejo das estratégias de rastreamento do CCU, pois promovem a cobertura da população de maior risco e apontam formas de reduzir as taxas de morbimortalidade por essa doença. ${ }^{23}$

Diante desse cenário, os enfermeiros são profissionais de referência para os cuidados nas diferentes fases do ciclo vital, em distintos territórios, como gestores e gerentes na tomada de decisões. Esses profissionais, com sua concepção ampliada do processo saúde, doença e cuidado, têm papel importante no que é desenvolvido ou não nos territórios e nas possibilidades que se abrem para novas práticas de saúde. ${ }^{24}$

\section{Limitações do estudo}

A pesquisa se limita a registros físicos nos quais dados importantes, como nível de escolaridade e situação socioeconômica, não estavam disponíveis ou estavam incompletos. Sugere-se, portanto, que outras pesquisas sejam realizadas abordando variáveis socioeconômicas.

\section{CONCLUSÃO}

Os resultados dos laudos analisados apontam para uma deficiência na qualidade dos exames indicada pela ausência de tecido glandular e metaplásico em número considerável de laudos, bem como para um grande número de mulheres com o exame Papanicolaou em atraso. Estes achados se tornam ainda mais relevantes diante dos diagnósticos de infecções sexualmente transmissíveis, como a tricomoníase, e de lesões precursoras do câncer do colo uterino, como lesão intraepitelial escamosa de alto grau. A presença destes resultados deve motivar a busca ativa imediata das respectivas pacientes a fim de quebrar a cadeia de transmissão de IST, evitar complicações advindas destas e tratar lesões no colo do útero detectadas precocemente, a fim de reduzir as chances de progressão.

\section{Contribuições do estudo para a prática}

Este estudo poderá sensibilizar profissionais sobre a necessidade da implementação de busca ativa de pacientes com este exame em atraso, especialmente das que apresentam alguma IST ou alteração celular prémaligna ou maligna.

\section{Agradecimentos}

Esta pesquisa contou com Bolsa de estudos da Coordenação de Aperfeiçoamento de Pessoal de Nível Superior (CAPES) para o terceiro autor.

\section{Contribuição dos autores}

NSM, FJML, DSF, MCLPS, DCJ, LBS contribuíram para a concepção e desenho, análise e interpretação dos da dos, redação do artigo, revisão crítica, revisão final.

\section{REFERÊNCIAS}

1. Pan American Health Organization, World Health Organization. Controle integral do câncer do colo do útero. Guia de práticas essenciais [Internet]. Washington, DC: PAHO; 2016 [acesso em 21 abr 2019]. Disponivel em: http://iris.paho.org/xmlui/bitstream/ handle/123456789/31403/9789275718797-por.pdf?sequence=1EisAllowed=y

2. Bray F, Ferlay J, Soerjomataram I, Siegel RL, Torre LA, Jemal A. Global cancer statistics 2018: GLOBOCAN estimates of incidence and mortality worldwide for 36 cancers in 185 countries. CA Cancer J Clin [Internet]. 2018 [acesso em 21 abr 2019];68(6):394-424. Disponivel em: https://doi.org/10.3322/caac.21492

3. World Health Organization, International Agency for Research on Cancer. Cancer today [Internet]. Geneva: WHO; 2019 [acesso em 21 abr 2019]. Disponivel em: http://gco.iarc.fr/today/home

4. Brasil, Instituto Nacional de Câncer. Diretrizes brasileiras para o rastreamento do câncer do colo do útero [Internet]. 2o ed. Rio de Janeiro: INCA; 2016 [acesso em 21 abr 2019]. Disponivel em: https://www.inca.gov.br/sites/ufu.sti.inca.local/files//media/ document//diretrizesparaorastreamentodocancerdocolodoutero_2016_corrigido.pdf/livros/diretrizes-brasileiras-para-orastreamento-do-cancer-do-colo-do-utero-2a-edicao

5. Brasil, Ministério da Saúde. Controle dos cânceres do colo do útero e da mama [Internet]. 2o ed. Brasilia: Ministério da Saúde; 2013 [acesso em 21 abr 2019]. 124 p. Disponivel em: http://bvsms.saude.gov.br/bvs/publicacoes/controle_canceres_colo_ utero_2013.pdf 
6. Brasil, Ministério da Saúde. Portaria no 2.436, de 21 de setembro 2017. Aprova a Política Nacional de Atenção Básica, estabelecendo arevisão de diretrizesparaa organização daAtenção Básica, noâmbito doSistema Único deSaúde(SUS)[Internet]. 2017. [acesso em 20 jul 2019]. Disponivel em: http://bvsms.saude.gov.br/bvs/saudelegis/gm/2017/prt2436_22_09_2017.html 7. Oliveira ES de, Silva ÍF da, Araújo AJ de S, Santos MVS, Queiroz PES. A consulta de enfermagem frente à detecção precoce de lesões no colo do útero. Rev Enferm Contemp [Internet]. 2017 [acesso em 27 abr 2019];6(2):186-98. Disponivel em: http://dx.doi. org/10.17267/2317-3378rec.v6i2.1369

8. Chantziantoniou N, Donnelly AD, Mukherjee M, Boon ME, Austin RM. Inception and Development of the Papanicolaou Stain Method. Acta Cytol [Internet]. 2017 [acesso em 30 mai 2019];61(4-5):266-80. Disponivel em: https://doi.org/10.1159/000457827 9. Chrysostomou AC, Stylianou DC, Constantinidou A, Kostrikis LG. Cervical Cancer Screening Programs in Europe: The Transition Towards HPV Vaccination and Population-Based HPV Testing. Viruses [Internet]. 2018 [acesso em 23 abr 2019];10(12). Disponivel em: https://dx.doi.org/10.3390\%2Fv10120729

10. World Health Organization. Cancer control: knowledge into action [Internet]. Geneva: WHO; 2007 [acesso em 22 abr 2019 ]. Disponivel em: https://www.who.int/cancer/modules/en/

11. Lim AW, Landy R, Castanon A, Hollingworth A, Hamilton W, Dudding N, et al. Cytology in the diagnosis of cervical cancer in symptomatic young women: a retrospective review. Br J Gen Pract [Internet]. 2016 [acesso em 23 abr 2019];66(653):e871-9. Disponivel em: https://doi.org/10.3399/bjgpl6X687937

12. Bringel APV, Rodrigues MP de F, Vidal ECF. Análise dos laudos de Papanicolaou realizados em uma unidade básica de saúde. Cogitare Enferm [Internet]. 2012 [acesso em 22 abr 2019];17(4). Disponivel em: http://dx.doi.org/10.5380/ce.v17i4.30385

13. Maier SR de O, Nascimento BED, Petri AP, Santos T da S. Avaliação da cobertura do exame citopatolóico de colo uterino em uma unidade de saúde da família no norte de Mato Grosso. Rev Eletronica Gest Saúde [Internet]. 2014 [acesso em 22 abr 2019];5(4):2532-49. Disponivel em: http://periodicos.unb.br/index.php/rgs/article/view/1033

14. Arbyn M, Anttila A, Jordan J, Ronco G, Schenck U, Segnan N, et al. European Guidelines for Quality Assurance in Cervical Cancer Screening. Second Edition-Summary Document. Ann Oncol [Internet]. 2010 [acesso em 23 abr 2019];21(3):448-58. Disponivel em: https://dx.doi.org/10.1093\%2Fannonc\%2Fmdp471

15. Mori MA, Ribeiro C nunes de morais. Falhas no diagnóstico do câncer de colo uterino. 2015 [acesso em 23 abr 2019$]$;(11). Disponivel em: https://www.researchgate.net/publication/320475052_FALHAS_NO_DIAGNOSTICO_DO_CANCER_DE_ COLO_UTERINO

16. Silva DSM da, Silva AMN, Brito LMO, Gomes SRL, Nascimento M do DSB, Chein MB da C. Rastreamento do câncer do colo do útero no Estado do Maranhão, Brasil. Ciênc Saúde Coletiva [Internet]. 2014 [acesso em 22 abr 2019];19(4):1163-70. Disponível em: http://dx.doi.org/10.1590/1413-81232014194.00372013

17. Campos RS, Silva IC, Silva LRL, Pinto MLS, Mochel EG. Prevenção do câncer de colo uterino: achados citológicos e microbiológicos em mulheres e uma cidade do nordeste - Maranhão. Rev Ciênc Saúde [Internet]. 2010 [acesso em 23 abr 2019];12(1):45-8. Disponivel em: http://www.periodicoseletronicos.ufma.br/index.php/rcisaude/article/view/969

18. Gerk MA de S, Girata MY, Nunes CB, Freitas SLF de. Cobertura do exame preventivo de câncer de colo de útero em uma unidade de saúde da familia. Cogitare Enferm [Internet]. 2014 [acesso em 22 abr 2019];19(2). Disponivel em: http://dx.doi. org/10.5380/ce.v19i2.32221

19. Jeronimo CG da F, Moraes M do N. Análise dos resultados dos testes citopatológicos do colo uterino. J Nurs UFPE on line [Internet]. 2015 [acesso em 22 abr 2019];9(3):7510-5. Disponível em: https://periodicos.ufpe.br/revistas/revistaenfermagem/ article/view/10488

20. Teixeira GA, Fônseca CJB, Lopes TRG, Carvalho JBL, Andrade FB. Prevalence of vulvovaginitis identified in cytological exam. J Nurs UFPE on line [Internet]. 2008 [acesso em 17 mai 2019];126(6). Disponivel em: https://periodicos.ufpe.br/revistas/ revistaenfermagem/article/view/10644

21. Weber AV, Backes LTH. Análise retrospectiva de inflamações cervicovaginais causadas por agentes microbiológicos no sul do Brasil. Rev Sau Int [Internet]. 2016 [acesso em 17 mai 2019];9(7). Disponivel em: http://local.cnecsan.edu.br/revistas/index. php/saude/article/view/272/288

22. Chen Y, Xu Y, Wu D, Li Z, Zhang Z, Meng Y, et al. Survey of cervical cancer and precancerous lesion prevention knowledge within community health service centers in Shanghai, China. Medicine(Baltimore) [Internet]. 2019 [acesso em 23 jun 2019];98(15). Disponivel em: https://dx.doi.org/10.1097\%2FMD.0000000000015002

23. Ribeiro JC, Andrade SR de, Ribeiro JC, Andrade SR de. Health surveillance and pap test coverage: integrative review. Texto Contexto - Enferm [Internet]. 2016 [acesso em 26 abr 2019];25(4). Disponivel em: http://dx.doi.org/10.1590/010407072016005320015

24. Fortuna CM, Matumoto S, Mishima SM, Rodríguez AMMM, Fortuna CM, Matumoto S, et al. Enfermagem em Saúde Coletiva: desejos e práticas. Rev Bras Enferm [Internet].2019 [acesso em 26 abr 2019];72:336-40. Disponivel em: http://dx.doi. org/10.1590/0034-7167-2017-0632 